\title{
Primary percutaneous coronary
} intervention during ST elevation myocardial
infarction in prosthetic valve endocarditis: a
case report

Alfonso Campanile ${ }^{1 * \dagger}$ (D) Guido Tavazzi ${ }^{2+}$, Francesco Caprioglio ${ }^{3}$ and Fausto Rigo ${ }^{3}$

\begin{abstract}
Background: Infective endocarditis (IE) is still a condition with high mortality and morbidity, especially in the elderly, and in patients with prosthetic valves. The concept of "time-to-therapy" plays a key role for the prompt management of IE and related complications, and the currently available multimodality imaging may play a key role in this setting. Myocardial ischemia due to extrinsic coronary compression from an aortic abscess is an extremely rare condition where the optimal therapeutic strategy has not been defined yet. We present herein the first case of a patient with ST elevation myocardial infarction caused by an aortic root abscess treated with percutaneous stent implantation.

Case presentation: An 82-year-old woman with a history of atrial fibrillation, chronic renal failure, anemia and a bioprosthetic aortic valve replacement performed in 2014, was admitted to hospital with profound asthenia and a pyrexia of unknown origin. Because of high clinical suspicion of endocarditis, a trans-esophageal echocardiogram was performed. Empirical broad-spectrum antimicrobial therapy was initiated, followed by targeted treatment based on the results of blood cultures (Staphylococcus aureus). The echocardiogram did not show vegetations and the patient was managed conservatively. She suddenly deteriorated, due to an acute coronary syndrome (ACS) with anterior ST segment elevation. An urgent angiogram was performed, and extrinsic compression of the left coronary system, due to an aortic root abscess, was suspected. After discussion with the surgical team, percutaneous revascularization was attempted, aiming to restore satisfactory hemodynamics, in order to plan surgery. Unfortunately, the patient rapidly developed cardiogenic shock, with multi organ failure, and died in less than $24 \mathrm{~h}$.
\end{abstract}

Conclusions: Patients with fever, and significant risk factors for endocarditis, who develop ACS, need a prompt diagnostic work up, including trans-esophageal echocardiography. At present, the specific timing of echocardiographic follow-up and surgical intervention is still a matter of debate, and our case aims to highlight the importance of this aspect in the management of endocarditis, in order to avoid severe complications that adversely affect patient prognosis.

Keywords: AMI, $\mathrm{PPCl}$, Endocarditis, TTE/TEE, Elderly patients

\footnotetext{
* Correspondence: alfonsocampanile@hotmail.it

${ }^{\dagger}$ Equal contributors

'Department of Cardiology, Hospital "S. M. della Misericordia", Perugia, Italy

Full list of author information is available at the end of the article
} 


\section{Background}

Acute coronary syndrome (ACS) occurs in only $1-3 \%$ of patients with infective endocarditis and the most appropriate management is therefore unknown $[1,2]$.

The possible mechanisms responsible for myocardial ischemia in endocarditis are the presence of preexisting coronary artery disease, coronary emboli from aortic vegetations, obstruction of the coronary ostium due to a large vegetation, severe aortic insufficiency and external coronary artery compression [3-5]. The latter is a rare and lethal finding with few reported cases available in the medical literature [3, 5-14].

We report a case of primary percutaneous coronary intervention (pPCI) performed during ST segment elevation myocardial infarction (STEMI) in the setting of a bioprosthetic aortic root abscess compressing the left main coronary artery. In previous similar reports, a surgical intervention represented the main therapeutic approach $[5,8,13]$. We believe that sharing experiences can be of help in defining a common procedural strategy in such rare conditions.

\section{Case presentation}

An 82-year-old woman with a history of atrial fibrillation, chronic renal failure, anemia and a bioprosthetic aortic valve replacement performed in 2014, was admitted to hospital with profound asthenia and a pyrexia of unknown origin (PUO). No coronary lesions were detected at the time of aortic valve replacement. Clinical parameters and laboratory findings on admission are summarized in Table 1. The chest X-ray did not show

\section{Table 1}

\begin{tabular}{ll}
\hline Clinical parameters & \\
Temperature $\left({ }^{\circ} \mathrm{C}\right)$ & 38,2 \\
Blood pressure $(\mathrm{mmHg})$ & $100 / 50$ \\
Mean heart rate $(\mathrm{bpm})$ & 88 \\
Peripheral oxygen saturation in room air (\%) & 95 \\
Laboratory findings & \\
Hemoglobin $(\mathrm{g} / \mathrm{L})$ & 108 \\
Hematocrit $(\%)$ & 30,5 \\
Mean corpuscular volume $(\mathrm{MCV}, \mathrm{fl})$ & 89 \\
Platelet count $\left(\times 10^{9} / \mathrm{L}\right)$ & 162 \\
White cell count $\left(\times 10^{9} / \mathrm{L}\right)$ & 18,7 \\
International normalized ratio $(\mathrm{INR})$ & 1,41 \\
Creatinine $(\mu \mathrm{mol} / \mathrm{L})$ & 169,8 \\
Azotemia $(\mathrm{mmol} / \mathrm{L})$ & 43,6 \\
Potassium $(\mathrm{mmol} / \mathrm{L})$ & 3,5 \\
Total bilirubin $(\mu \mathrm{mol} / \mathrm{L})$ & 23,9 \\
C-reactive protein $(\mathrm{nmol} / \mathrm{L})$ & 916,2 \\
Procalcitonin $(\mu \mathrm{g} / \mathrm{L})$ & 0,93 \\
\hline
\end{tabular}

signs of consolidation. Samples of urine, blood and sputum were sent for microbiological screening. A transesophageal echocardiogram (TEE) was performed as part of the PUO diagnostic work-up [15] and showed the left ventricle was not dilated with normal global systolic function, moderate mitral and tricuspid regurgitation, normal bioprosthesic function but pronounced aortic root thickening (Fig. 1 and Additional file 1: Video S1). Because of high clinical suspicion of endocarditis, empirical broad spectrum antimicrobial therapy was initiated, followed by targeted treatment (clyndamicin $600 \mathrm{mg}$ three times daily) based upon the results of blood cultures (Staphylococcus aureus). The initial patient response was satisfactory. She became afebrile and the inflammatory markers improved. A conservative strategy was planned. During her first week in hospital she underwent a routine transthoracic echocardiogram (TTE) and a second TEE was also repeated, 7 days later, with no new findings. Due to the stable clinical conditions, the initial management plan remained unchanged. It was aimed to complete 1 month of antibiotic therapy before performing a new TEE. However, almost 1 month after her admission, a new rise in her inflammatory markers and temperature was observed associated with clinical deterioration. $24 \mathrm{~h}$ later the patient developed central chest discomfort and an ECG showed new ST segment elevation in the anterior precordial leads (Fig. 2a and b). Immediate coronary angiography was performed. A severe narrowing, followed by complete occlusion of the left anterior descending coronary artery (LAD) and a critical, long tubular stenosis of the proximal left circumflex artery (LCx), was detected (Fig. 2c, Additional file 2: Video S2). The interventional cardiologist suspected that there was extrinsic compression of the coronary arteries from the aortic root abscess (Additional file 3: Video S3). The surgical team was involved, however due to the

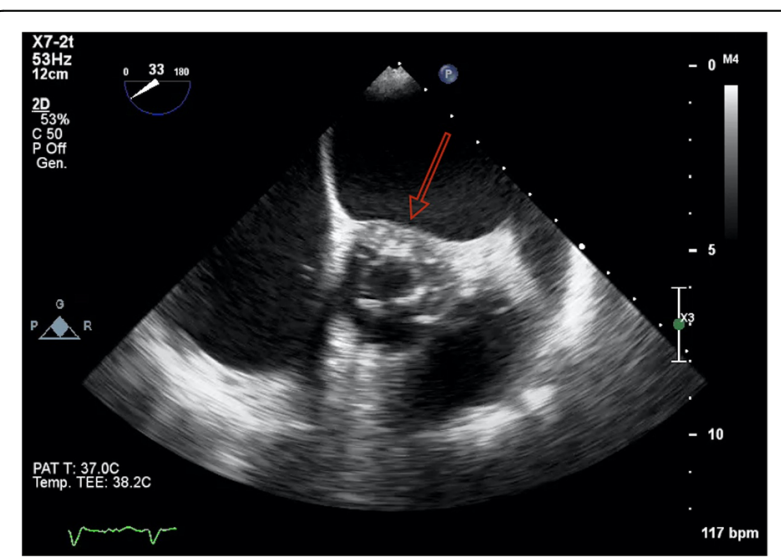

Fig. 1 Trans-esophageal short axis view of the aorta showing the aortic bioprosthesis in a correct position, and a pronounced aortic root thickening (marked with the red arrow in the picture) 


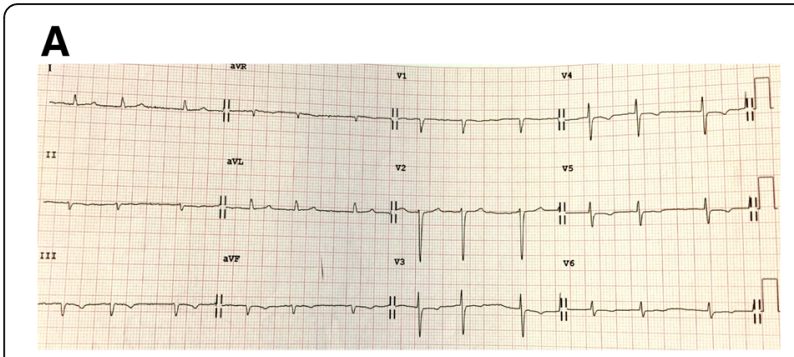

B

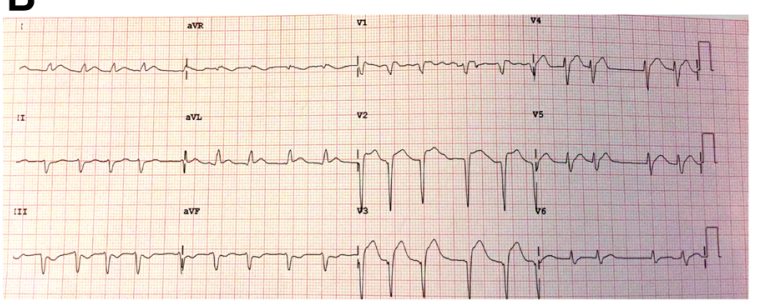

C

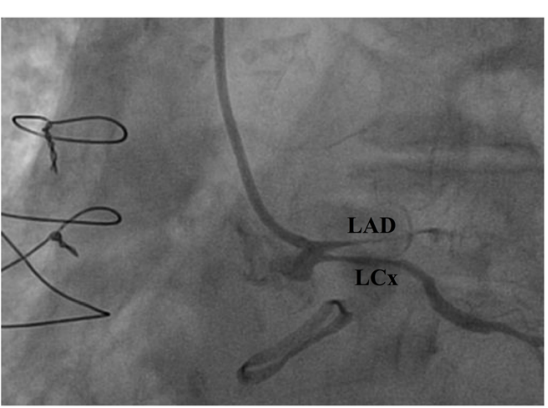

Fig. $\mathbf{2}$ a, $\mathbf{b}$ and $\mathbf{c}$ (from the top to the bottom): a basal ECG showing atrial fibrillation with T-wave inversion in the inferior and anterolateral leads. b ECG acquired during chest pain showing anterior ST elevation. c The left coronary angiogram, showing occlusion of the proximal LAD and a long, tubular stenosis of the proximal LCX

unstable hemodynamic condition (blood pressure: 80/ $50 \mathrm{mmHg}$, heart rate-HR: $110 \mathrm{bpm}, \mathrm{pO} 2: 8 \mathrm{kPa}$ in high flow oxygen treatment), it was decided to attempt percutaneous treatment. Two bare metal stents (BMS-Rebel Boston Scientific ${ }^{\circ}$ ) were implanted respectively in the proximal segment of the LAD $(3.0 * 24 \mathrm{~mm})$ and the LCx $(3.5 * 20 \mathrm{~mm})$ (Fig. 3, Additional files 2, 3, 4, 5, 6, 7, 8 and 9: Videos S2-S9).

On admission to the coronary care unit the patient was in cardiogenic shock requiring inotropes, vasopressors and non-invasive ventilation. The biochemical results showed a white blood cell count (WBC) of $25,7 \times$ $10^{9} / \mathrm{L}$, creatine kinase-MB fraction $184 \mu \mathrm{g} / \mathrm{L}$, and Tropo$\operatorname{nin} \mathrm{I}(\mathrm{TnI})>70.000 \mu \mathrm{g} / \mathrm{L}$. A transthoracic echocardiogram was performed (Fig. 4, Additional files 10, 11, 12, 13 and 14: Videos S10-S14) with evidence of severe biventricular dysfunction, severe mitral and tricuspid regurgitation, and significant tissue damage in the aortic prosthetic region, where a cavity was also identified, suggesting an abscess. Despite intensive treatment the patient died within $24 \mathrm{~h}$.

\section{Discussion}

We describe a rare complication of prosthetic valve endocarditis: an aortic root abscess causing external coronary artery compression and acute myocardial infarction. Although a few reports of myocardial infarction during infective endocarditis involving a prosthetic valve exist in the literature, our case shows an unusual complication and treatment strategy.

Prosthetic valve endocarditis is associated with a high in-hospital mortality (more than 40\%) [16, 17] and when complicated by an ACS, the morbidity and mortality rates further increase [18]. In the management algorithm presented by Manzano et al., when a patient presents with ACS and suspected endocarditis, a TEE should be performed and angiography, with percutaneous reperfusion, should only be considered if a ST segment elevation is present on the ECG in the absence of periannular complication [1]. In our case, an immediate coronary angiogram was performed because ST elevation myocardial infarction, complicated by haemodynamic instability, was evident, while the abscess
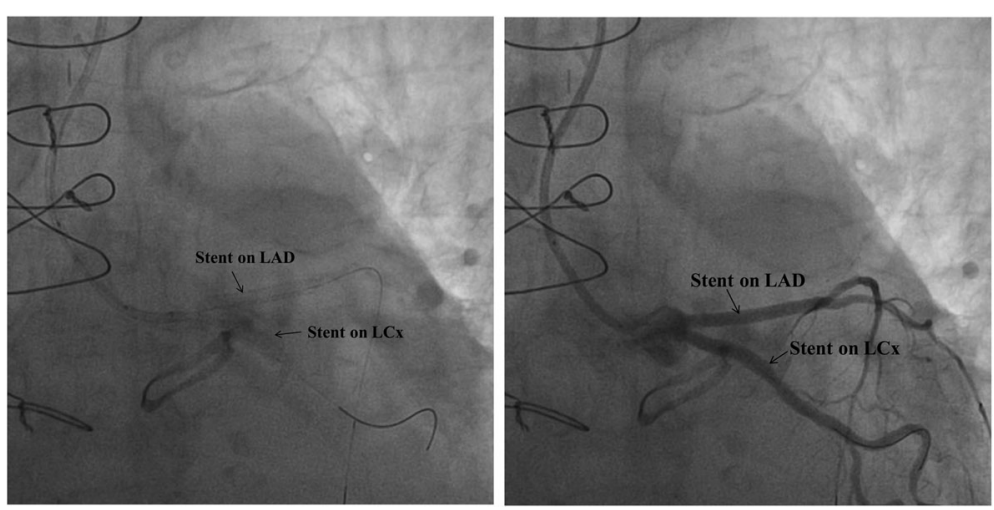

Fig. 3 The stent implantation procedure in the left coronary system before (on the left) and after (on the right) contrast injection; stents and coronary vessels involved are marked with black arrows 

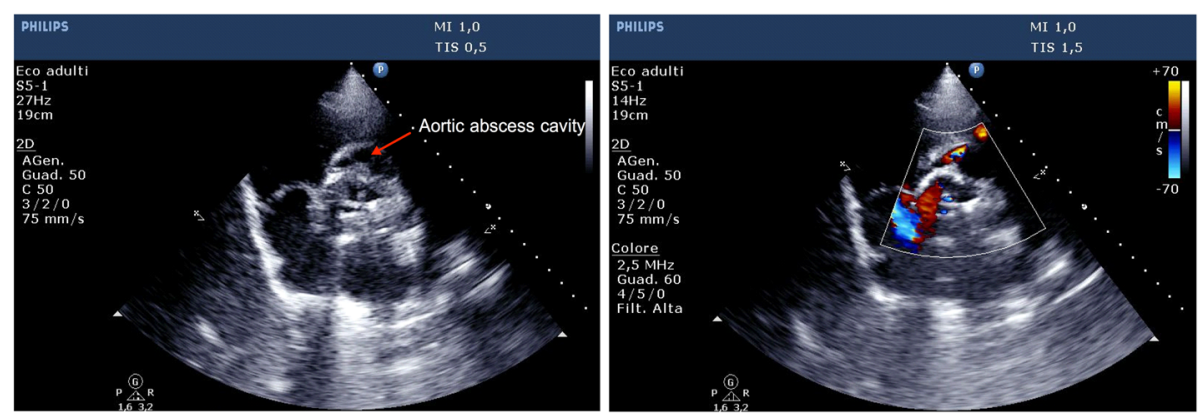

Fig. 4 Trans-thoracic short axis view of the aorta in bi-dimensional (on the left) and color mode representation (on the right), showing a cavity around the aortic root, highly suspicious for a peri-prosthetic abscess

cavity was only suspected. The hypothesis of external coronary compression due to periannular complication was, indeed, only formulated at the time of the invasive procedure due to the specific angiographic pattern (diffuse narrowing of the coronary arteries with severe stenosis of the proximal segments) along with the peculiar clinical presentation [19]. However, an erosion/perforation of the abscess into left coronary artery, could not be definitely ruled out. Some images, indeed, showed enrichment of the contrast medium in the abscess during angiography (Additional files 2 and 3: Video S2 and S3).

After a discussion with the surgical team, a two-step approach, with percutaneous revascularization first, aiming for restoration of stable hemodynamics, followed by a surgical procedure, was attempted, similar to the case described by Sugi K et al. [20].

The outcome in this setting is strongly affected by several different variables (grade of inflammatory response, pathogen causing endocarditis, sepsis dissemination, site of coronary obstruction and the grade of valve dysfunction) [20] and, unfortunately, our patient rapidly developed multi organ failure.

\section{Conclusions}

Considering the paucity of data available, the management of each patient presenting with ST elevation and valve/prosthetic endocarditis should be individualized and a multidisciplinary discussion should be advocated.

Patients with fever, and significant risk factors for endocarditis, who develop ACS, need a prompt diagnostic work up, including trans-esophageal echocardiography. The specific timing of echocardiographic followup and surgical intervention is still a matter of debate $[17,21]$, and our case aims to highlight the importance of this aspect in the management of endocarditis, in order to avoid severe complications that adversely affect patient prognosis.

\section{Additional files}

Additional file 1: Video S1. (Trans-esophageal short axis view of the aorta): the clip shows a normal opening of the aortic bioprosthesis and a pronounced aortic root thickening. (MOV $702 \mathrm{~kb}$ )

Additional file 2: Video S2. Clip of the interventional procedure performed to establish a satisfactory flow on the left coronary system. (Left coronary angiography): the diagnostic left coronary angiogram showing a severe narrowing, followed by complete occlusion of the left anterior descending coronary artery (LAD) and a critical, long tubular stenosis of the proximal left circumflex artery (LCX). (MOV $1760 \mathrm{~kb}$ )

Additional file 3: Video S3. Clip of the interventional procedure performed to establish a satisfactory flow on the left coronary system. (Ascending aortic angiography): the ascending aortic angiogram showing a possible, extrinsic compression, of the coronary arteries from an aortic root abscess. The clip also shows enrichment of the contrast medium in the abscess during angiography so that, an erosion/perforation of the abscess into left coronary artery, can't be definitely ruled out. (MOV 3090 kb)

Additional file 4: Video S4. Clip of the interventional procedure performed to establish a satisfactory flow on the left coronary system. (Wires advancement in the left coronary system): the clip shows the wires that have been introduced in the LAD and LCX. (MOV $3720 \mathrm{~kb}$ )

Additional file 5: Video S5. Clip of the interventional procedure performed to establish a satisfactory flow on the left coronary system. (After only balloon angioplasty): video showing the result soon after POBA. (MOV $3030 \mathrm{~kb}$ )

Additional file 6: Video S6. Clip of the interventional procedure performed to establish a satisfactory flow on the left coronary system. (Stent deployment in the LAD). (MOV $1130 \mathrm{~kb}$ )

Additional file 7: Video S7. Clip of the interventional procedure performed to establish a satisfactory flow on the left coronary system. (Post stent implantation in the $L A D$ ): videos showing the stent deployment in the LAD and the result of the procedure. (MOV $1890 \mathrm{~kb}$ )

Additional file 8: Video S8. Clip of the interventional procedure performed to establish a satisfactory flow on the left coronary system. (Stent deployment in the LCX artery). (MOV $511 \mathrm{~kb}$ )

Additional file 9: Video S9. Clip of the interventional procedure performed to establish a satisfactory flow on the left coronary system. (Final result): videos showing the stent deployment in the LCX and the final procedural result. (MOV $2800 \mathrm{~kb}$ )

Additional file 10: Video S10. Clip of the trans-thoracic examination. (Parasternal long axis view): evidence of severe left ventricle dysfunction. (M4V $371 \mathrm{~kb}$ )

Additional file 11: Video S11. Clip of the trans-thoracic examination. (Parasternal long axis view with color): the clip shows a significant tissue damage in the aortic prosthetic region, where a cavity is also identified, suggesting an abscess. (M4V $328 \mathrm{~kb}$ ) 
Additional file 12: Video S12. Clip of the trans-thoracic examination. (Parasternal short axis of the aortic valve without and with color mode representation): different view of the significant tissue damage in the aortic prosthetic region. (MP4 $6072 \mathrm{~kb}$ )

Additional file 13: Video S13. Clip of the trans-thoracic examination. (Apical four chamber view without and with color mode representation): evidence, respectively, of severe biventricular dysfunction, severe TR and MR. (MP4 $8888 \mathrm{~kb})$

Additional file 14: Video S14. Clip of the trans-thoracic examination. (Subcostal view): view showing a dilated inferior vena cava and right pleural effusion. (M4V $367 \mathrm{~kb}$ )

\section{Abbreviations}

ACS: Acute coronary syndrome; BMS: Bare metal stent; IE: Infective endocarditis; ECG: electrocardiogram; LAD: left anterior descending coronary artery; LCx: left circumflex artery; POBA: plain old balloon angioplasty; TR: tricuspid regurgitation; MR: mitral regurgitation; $\mathrm{PPCl}$ : Primary percutaneous coronary intervention; PUO: Pyrexia of unknown origin; STEMI: ST segment elevation myocardial infarction; TEE: Trans-esophageal echocardiogram; TTE: Transthoracic echocardiogram; WBC: White blood cell count

\section{Acknowledgements}

The authors thank Dr. Ada Cutolo and Dr. Guerrino Zuin of the Cardiology Department in Mestre, Italy, for their contribution in the data collection process.

\section{Funding}

Not applicable.

\section{Availability of data and materials}

All relevant data supporting the conclusions of this article are contained within the article.

\section{Authors' contributions}

AC was directly involved in the patient management during her admission in coronary care unit and personally performed the trans-thoracic and trans-esophageal echocardiograms reported in the paper. He was a major contributor in writing the manuscript, and also performs all duties of the corresponding authors; GT made substantial contributions to conception and design of the work. He also helped with writing and drafting the manuscript. FC performed the invasive procedure. FR was involved in the patient management and also in drafting the manuscript and critical revision for important intellectual content. He made substantial contributions to analysis and interpretation of data. All authors read and approved the final article.

\section{Competing interest}

The authors declare that they have no competing interests.

\section{Ethics approval and consent to participate}

Not applicable.

\section{Consent for publication}

Written consent was obtained from the patient's relatives for the publication of this case report.

\section{Publisher's Note}

Springer Nature remains neutral with regard to jurisdictional claims in published maps and institutional affiliations.

\section{Author details}

'Department of Cardiology, Hospital "S. M. della Misericordia", Perugia, Italy. ${ }^{2}$ Department of Anaesthesia, Intensive Care and Pain Therapy, Fondazione Policlinico San Matteo IRCCS, University of Pavia, Pavia, Italy. ${ }^{3}$ Department of Cardiology, Dell'Angelo Hospital, Mestre, Italy.
Received: 21 June 2017 Accepted: 18 January 2018

Published online: 09 February 2018

\section{References}

1. Manzano MC, Vilacosta I, San Roman JA, Aragoncillo P, Sarria C, Lopez D, et al. Acute coronary syndrome in infective endocarditis. Rev Esp Cardiol. 2007; 60(1):24-31. PubMed PMID: 17288952. Sindrome coronario agudo en la endocarditis infecciosa. spa

2. Attias D, Messika-Zeitoun D, Wolf M, Lepage L, Vahanian A. Acute coronary syndrome in aortic infective endocarditis. Eur J Echocardiogr. 2008;9(6):727-8. PubMed PMID: 18579492 eng

3. Schlaifer JD, Martin TD, Hill JA, Kerensky RA. Coronary artery obstruction caused by perivalvular abscess in aortic valve endocarditis. Am Heart J. 1996;131(2):413-6. PubMed PMID: 8579047. eng

4. Khan JA, Panwar Z, Mujtaba F, Shah K. Infective endocarditis: a rare cause of acute coronary syndrome. J Coll Physicians Surg Pak. 2012;22(4):248-9. PubMed PMID: 22482383. eng

5. Atik FA, Campos VG, da Cunha CR, de Oliveira FB, Otto ME, Monte GU. Unusual mechanism of myocardial infarction in prosthetic valve endocarditis. Int Med Case Rep J. 2015;8:111-6. PubMed PMID: 26045678. eng

6. Cripps T, Guvendik L. Coronary artery compression caused by abscess formation in infective endocarditis. Int J Cardiol. 1987;14(1):99-102. PubMed PMID: 3804511. eng

7. Parashara DK, Jacobs LE, Kotler MN, Yazdanfar S, Spielman SR, Janzer SF, et al. Angina caused by systolic compression of the left coronary artery as a result of pseudoaneurysm of the mitral-aortic intervalvular fibrosa. Am Heart J. 1995;129(2):417-21. PubMed PMID: 7832123. eng

8. Zoffoli G, Gherli T. Images in cardiovascular medicine. Periaortic valve abscess presenting as unstable angina. Circulation. 2005;112(13):e240-1. PubMed PMID: 16186428. eng

9. Cowan SW, Fiser SM, Albrecht M, Fifer M, Vlahakes GJ, Madsen JC. Management of coronary artery compression caused by recurrent aortic root abscess. J Card Surg. 2008;23(3):195-7. PubMed PMID: 18435630. eng

10. Esen AM, Acar G, Alizade E. Prosthetic aortic valve abscess producing left main coronary artery occlusion in a patient with type IV dual left anterior descending coronary artery. J Invasive Cardiol. 2011;23(1):E233-5. PubMed PMID: 21183771. eng

11. Jose J, Narayanan K, Chacko ST, George OK. Infective endocarditis presenting with ST segment elevation. J Heart Valve Dis. 2011;20(4):468-70. PubMed PMID: 21863663. eng

12. Misuraca L, De Caro F, De Carlo M, Barzaghi C, Scioti G, Minzioni G, et al. Left main compression by an aortic root abscess. J Cardiovasc Med (Hagerstown). 2012;13(3):207-8. PubMed PMID: 22306781. eng

13. Harinstein ME, Marroquin OC. External coronary artery compression due to prosthetic valve bacterial endocarditis. Catheter Cardiovasc Interv. 2014 83(3):E168-70. PubMed PMID: 24660223. eng

14. Jenny BE, Almanaseer Y. Aortic valve endocarditis complicated by STelevation myocardial infarction. Tex Heart Inst J. 2014;41(6):668-70. PubMed PMID: 25593539. eng

15. Hersch EC, Oh RC. Prolonged febrile illness and fever of unknown origin in adults. Am Fam Physician. 2014;90(2):91-6. PubMed PMID: 25077578. eng

16. Hoen B, Duval X. Clinical practice. Infective endocarditis. N Engl J Med. 2013, 368(15):1425-33. PubMed PMID: 23574121. eng

17. Habib G, Lancellotti P, Antunes MJ, Bongiorni MG, Casalta JP, Del Zotti F, et al. 2015 ESC guidelines for the management of infective endocarditis: the task force for the management of infective endocarditis of the European Society of Cardiology (ESC). Endorsed by: European Association for Cardio-Thoracic Surgery (EACTS), the European Association of Nuclear Medicine (EANM). Eur Heart J. 2015;36(44):3075128. PubMed PMID: 26320109

18. Nishimura RA, Carabello BA, Faxon DP, Freed MD, Lytle BW, O'Gara PT, et al. ACC/AHA 2008 guideline update on valvular heart disease: focused update on infective endocarditis: a report of the American College of Cardiology/ American Heart Association task force on practice guidelines endorsed by the Society of Cardiovascular Anesthesiologists, Society for Cardiovascular Angiography and Interventions, and Society of Thoracic Surgeons. J Am Coll Cardiol. 2008;52(8):676-85. PubMed PMID: 18702976. eng

19. Clarke NR, Forfar JC. Aortic root abscess presenting as unstable angina due to extrinsic compression of the left coronary artery. Postgrad Med J. 2002: 78(917):168-9. PubMed PMID: 11884701. Pubmed Central PMCID: 1742294 
20. Sugi K, Nakano S, Fukasawa Y, Maruyama R, Tanno J, Senbonmatsu T, et al. Percutaneous coronary intervention for septic emboli in the left main trunk as a complication of infective endocarditis. Heart Lung Circ. 2015;24(11): e176-9. PubMed PMID: 26251314. eng

21. Lalani T, Chu VH, Park LP, Cecchi E, Corey GR, Durante-Mangoni E, et al. In-hospital and 1-year mortality in patients undergoing early surgery for prosthetic valve endocarditis. JAMA Intern Med. 2013;173(16):1495-504. PubMed PMID: 23857547

Submit your next manuscript to BioMed Central and we will help you at every step:

- We accept pre-submission inquiries

- Our selector tool helps you to find the most relevant journal

- We provide round the clock customer support

- Convenient online submission

- Thorough peer review

- Inclusion in PubMed and all major indexing services

- Maximum visibility for your research

Submit your manuscript at www.biomedcentral.com/submit 